\title{
Research on the Video Advertising Detection Based on LS-SVM
}

\author{
Xiao-ling Lan ${ }^{1}$ and Shutuan Zhang ${ }^{2}$ \\ 1. The Art College, Lu Dong University, Yantai, China, 264025 \\ 2. Department of Control Engineering, NAAU, Yantai, China, 264001 \\ 848446636@qq.com
}

\begin{abstract}
Aimed to the difficult problem of detection information of video advertising from the massive video data, the novelty detection method of the video advertising based on the least squares support vector machine (LS-SVM) algorithm is introduced after analyzing and studying the feature of video advertising. The detection of the abrupt change shot and the gradual change shot in video data is realized by using color histogram algorithm, and the classification of the shot is achieved by using the particle swarm optimization and least squares support vector machine algorithm. The experiments show that the method based on the color histogram algorithm and LS-SVM algorithm has better Precision and recall, and can realize the accuracy detection of the video advertising.
\end{abstract}

Keywords: video advertising detection, color histogram, shot segmentation, least squares support vector machine

\section{Introduction}

With the continuous development of the multimedia technology and network technology, the video has become one of popular media forms. Video advertising plays an important role in the course of daily information dissemination as the main carrier of commercial information. The public's viewpoints about video ads are divided: (1) For ordinary video browsing personnel, they are not interested in video ads, who hope that the ads will be removed; (2) persons interested advertising or advertising practitioners, hope to quickly locate video ads and find the information from the huge repository of information in the shortest possible time, thereby to improve their work efficiency ; (3) Advertisers hope to quickly browse to their video ads to evaluate the advertising effect. However, faced with a huge video files and rich amounts of information, how to fast and efficiently storage, classify, retrieve and use those relevant video advertising is an urgent problem. Therefore, the rapid detection of video advertising has become a hot research. For the rapid detection of video advertising, we propose a color histogram and video ads detection method of Least Squares Support Vector Machine (LS-SVM) based on the shot segmentation method using color histogram lens edge detection method, then using LSSVM to classify the lens, the lens finally combined to get the full ad video advertising clips, enabling automatic rapid positioning video advertising segment [1-2].

\section{The Character of Video Advertising}

The video data is a two-dimensional flow sequence, which is composed of a series of image frames, and the picture frame is the smallest unit of video. Video data is a very important kind of vision information source, which contains not only the content of static images, also contains information of target motion in the scene varied and information of the objective world with time. Video data has spatial attribute and time characteristics, so a video is represented by a three-dimensional array $E(x, y, t)$ including of time and space coordinate. Then the color or brightness of each pixel can be expressed as a function on 
the XY plane $E(x, y, t)$. A video tells a complete story included by a series of event plots which describes a specific event, and each plot is composed of several scenes. Each scene contains a sub event or an action, and it contains multiple lenses with related content.

The continuous movement shot in a space scene corresponding to a lens which is indicated by a series of successive images. So the lens is composed of a lot of video frames. The structure of video data is shown in Figure 1.

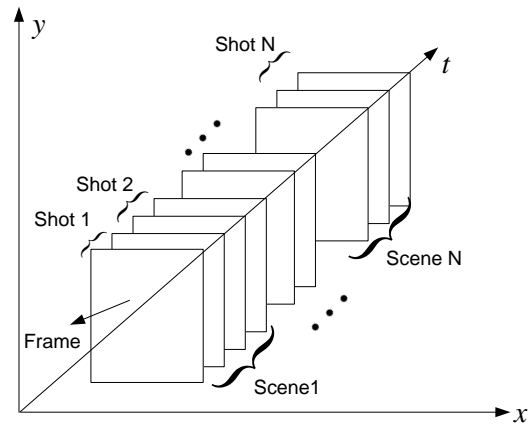

Figure 1. The Sketch of Video Information

Video advertising has its own characteristics. It need to transfer the business information within a specified time through a variety of expressions and shooting techniques to the mass, and brings the audience a strong stimulus of information in a short time, so compared to the ordinary program video, video advertising has a significant difference in the expression. The theme of a Video advertising is the core message to convey. So we may depart from the subject of video advertising, and progressively analyze the unique structural features of video advertising, then we can achieve their detection and localization by analyzing the elements of video advertising In order to give viewers strong message stimulation in the shortest time, video ads will switch lens very fast. The lens is an essential part of the video, the accuracy of shot segmentation and determines the effect of the detection system in the very great degree. The structure chart of video advertising is shown in Figure 2.

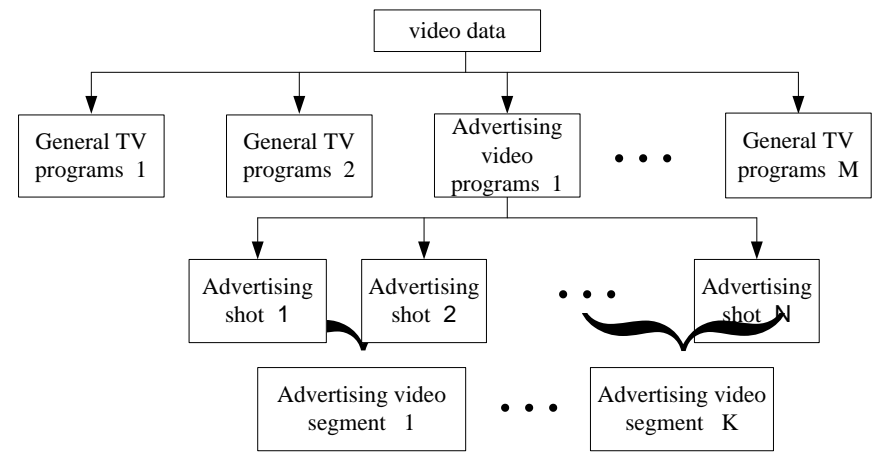

Figure 2. The Structure Chart of Video Advertising

\section{Video Advertising Shot Detection Method $[1,3]$}

The transition way between two successive shots is called shot switch [4], the transition point of the shots that is separate and convergence of video sequences between two different lenses, is achieved on the director switcher or stunt generator. Usually the basic types of shots switching include mutation (abrupt change) and gradient (gradual change). Mutation refers to a lens directly switching to the next one without delay. The 
shear of lens can make the plot and action of videos directly jump, and bring a light and agile feeling; gradient is added to some space and time editing effects. A lens forward gradually converts to the next one without obvious visual jumps. Due to the different editing modes, common types of gradient include ablation (dissolve), fade (fade in / out), draw variable (wipe).

The characteristics of the video advertising determine its expression, compared to ordinary program video, advertising video uses more lens conversion. The mutation is the main method of camera lens and also is the usual form of a scene change; gradient lens is mainly used to produce the effects of advertising video, especially in the well-known brands of cosmetics advertising to render certain cosmetic effect.

Shot boundary is formed by two lens to switch. The transformation of the boundary is caused by the video content to cut off. Through directly connecting two lenses without using any video editing effects to complete the shear, which introduces the visual difference of frame image between two lens. Therefore, the lens mutation is due to the sudden change of visual content between the last frame image of the lens forward and the first frame of the adjacent lens. At present, the detection method of commonly used lens mutation is to use the discontinuity between the lens, and to adopt a similar image segmentation method based on boundary. The basic steps of this method are firstly to extract characteristic information, and calculate the characteristic value difference between two frames, and then compare the difference to a certain threshold. If it is greater than the threshold, it is considered that the two adjacent frames respectively belong to different lenses, which also detects shot boundary; otherwise think that this two adjacent frames belong to the same lens. So, the selection of visual features and distance measurement function and the selection of threshold value are the key for detection of lens mutation.

The Grayscale and brightness value of each pixel is the elements to directly reflect the visual content of images. SO the simplest method to measure the visual difference between two adjacent frames is to calculate the gray scale and brightness value of all pixels in the adjacent frames which also has a drawback that is sensitive to the motion of camera and objects. When the motion is large, the difference of adjacent frames often exceeds a set threshold, which results in error in lens detection. To overcome this shortcoming, it is necessary to base on the statistical information of pixels to calculate the gray scale difference of adjacent frames. So the histogram comparison algorithm is proposed. The adjacent frames of the same scene generally have the same visual elements in whole situation because a lens is a reflection of the shooting action of a camera. Namely adjacent frames of the same lens have similar gray distribution. The reaction on the histogram is: the smaller difference adjacent frame exists between the same shot; the larger difference exists between the frames in different lens .Obviously, there is also a situation in theory. Though visual content is not similar, the difference is small on the histogram. But in real video sequences, the probability of the occurrence of such a situation is very small. Relatively speaking, the method based on a histogram is the most simple and effective method.

Currently, the detection method is different according to different shot edge. For example pixel difference method, statistic method(e.g. mean and variance within the window), image features (such as image edges), histogram method, compressed domain detection method, regional block method, spatial and temporal flow method and other methods are applied in the appropriate lens detection. Compared to news, television programs, etc., video advertising has its own unique characteristics, such as the frame has bright and varied colors, the frequency of shear lens is higher. So the color or grayscale histogram [5-6] difference between shear detection methods can get a good shot detection effect.

The histogram is an effective tool for reflecting the probability of the luminance distribution of a grayscale image, the color histogram of the color image also reflects the 
distribution of color information in the space of the color, so the key determination is extraction of color feature using color histograms. The color feature of fame images may be the color distribution ratio or the spatial distribution, etc. The shear shot detection algorithm based on color histogram adopt the distribution of the color scale to obtain color features, color image color histogram is the most universal representation of image color characteristic. The basic idea of the algorithm is attributed to differences in image color histogram for the gap between, which will convert the detection of shear lens to calculate the distance of color histogram. Therefore, the color histogram method measures the distance between the image color histogram to measure the similarity between images.

Color histogram is a statistical probability method, which satisfies the number of pixels of image, and expresses the result in the form of a histogram of the clip. First, calculate the brightness or color histogram of the image based on the histogram method and then different methods can be used to compare two frames before and after the image histogram statistics, the most direct way is to use normalized to compare the color histogram the distance between each component of figure D:

$$
D=\frac{\sum_{k=0}^{K-1}\{H[f(x, y, t), k]-H[f(x, y, t+1), k]\}^{2}}{\sum_{k=0}^{K-1} H[f(x, y, t+1), k]}
$$

If the $\mathrm{D}$ is greater than two images in a pre-determined threshold value, then the lens is generally considered handover. Because the presence of an object or movement of the lens between the before and after frames is generally very similar to the histogram, so using the motion can effectively reduce the influence due to the switching of the lens or movement of the subject. At the same time, in order to reduce the affect of local lighting and target motion, the image can be divided into small pieces; the above equation is calculated separately for each piece. Then some blocks will be deleted that the difference value is large and the other is be considered. But some scenes with different objectives which are approximate grayscale or color histogram distribution are likely to cause missed. In the shot segmentation stage, the lens is an essential part of the video. So the accuracy of shot segmentation will determines the effect of the detection system to a large extent. Color histogram method has higher detection rate in video ads lens detection, so this paper uses the method of video ads for shot detection.

\section{The Video Advertising Shot Detection Based on the LS-SVM Algorithm}

A. Least Squares Support Vector Machine algorithm

Support Vector Machine (SVM) is a machine algorithm based on statistical learning theory on the principle of structural risk minimization. For the principle of SVM classification as shown in Figure 3, "○" and " " point represent two types of samples, $x$ is the optimal hyper plane, respectively, $x_{(1)} 、 x_{(2)}$ are the closest simples to classification in over two class and is parallel to optimal hyper plane of the classification plane. The distance between them is the class interval. 


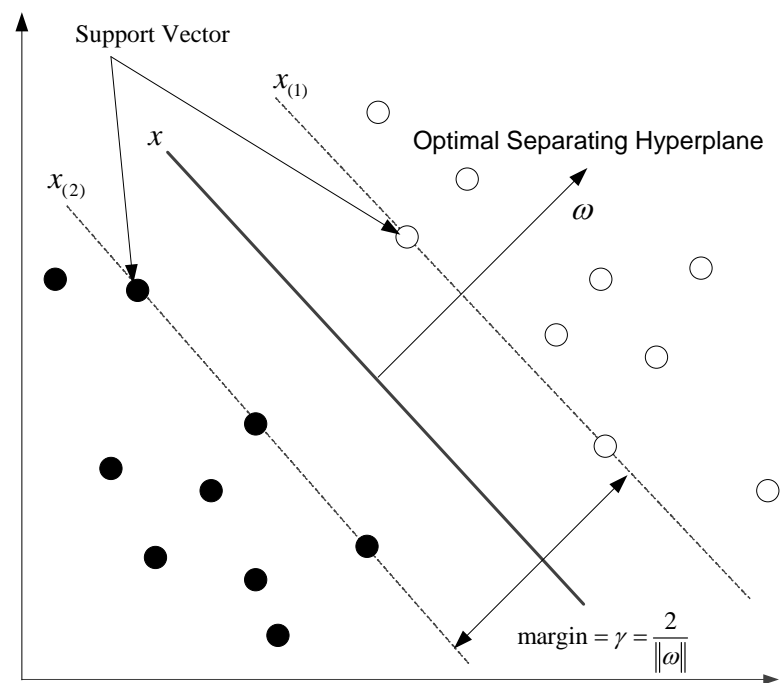

Figure 3. The Sketch of Separating Hyperplane

The algorithm of Least Squares Support Vector Machine, proposed by Suykens and Vandewalb in 1999, is an improved algorithm based on structural risk minimization rule. LS-SVM is the form of the support vector machine [7-8] with two loss function. In LSSVM, the non-sensitive loss function is replaced by quadratic loss function and the inequality constraints are replaced by equality constraints.

The minimum object function and constraint condition has been modified as follows:

$$
\min J(\omega, \xi)=\frac{1}{2}\|\omega\|^{2}+\frac{\gamma}{2} \sum_{i=1}^{N} \xi_{i}^{2}
$$

s.t. $\quad y_{i}\left[\omega^{T} \phi\left(x_{i}\right)+b\right]=1-\xi_{i}, \quad i=1,2, \cdots, N$

Where $J(\omega, \xi)$ is structure risk, $\gamma$ is the regularization parameter, determining the trade-off between the fitting error minimization and smoothness. $\xi$ is a slack variable, which ensures classification validity under linear non-separable case, $\phi$ is the nonlinear mapping function which transform the nonlinear problem into a linear problem of higher dimension feature space. The function $y_{k}$ is the value 1 , when the $x_{k}$ belong to the type I , otherwise, the function $y_{k}$ is the value -1 , when the $x_{k}$ belong to the type $I$, that is to say,

$$
y_{k}=\left\{\begin{array}{ll}
1 & x_{k} \text { belong to type I } \\
-1 & x_{k} \text { belong to type II }
\end{array} .\right.
$$

In this transforming space, we can acquire the optimal hyperplane. The Lagrangian function is introduced in order to solve the optimal problem.

$$
L(\omega, b, \xi, \alpha)=\frac{1}{2} \omega^{T} \omega+\frac{\gamma}{2} \sum_{i=1}^{N} \xi_{i}^{2}-\sum_{i=1}^{N} \alpha_{i}\left\{y_{i}\left[\omega^{T} \phi\left(x_{i}\right)+b\right]-1+\xi_{i}\right\}
$$

Where $\alpha_{i}$ is the Lagrange multipliers. Considering the optimization conditions by Karush-Kuhn-Tucker (KKT),

$$
\frac{\partial L}{\partial \omega}=0, \frac{\partial L}{\partial b}=0, \frac{\partial L}{\partial \xi_{i}}=0, \frac{\partial L}{\partial \alpha_{i}}=0
$$

The optimal condition about equation (3) can be obtained as following: 


$$
\left\{\begin{array}{l}
\omega=\sum_{i=1}^{N} \alpha_{i} y_{i} \phi\left(x_{i}\right) \\
\sum_{i=1}^{N} \alpha_{i} y_{i}=0 \\
\alpha_{i}=\gamma \xi_{i} \\
y_{i}\left[\omega^{T} \phi\left(x_{i}\right)+b\right]-1+\xi=0
\end{array}\right.
$$

Where $i=1,2, \cdots, N$.

Eliminating the parameter $\omega$ and $\xi$ in equation (5), the equation can be rewritten as:

$$
\left[\begin{array}{lr}
0 & Y \\
Y^{T} & Q+\frac{I}{\gamma}
\end{array}\right]\left[\begin{array}{l}
b \\
\alpha
\end{array}\right]=\left[\begin{array}{l}
0 \\
1_{V}
\end{array}\right]
$$

Where $Y=\left[y_{1}, \cdots, y_{N}\right], 1_{V}=[1, \cdots, 1]^{T}, \xi=\left[\xi_{1}, \cdots, \xi_{N}\right]^{T}, \alpha=\left[\alpha_{1}, \cdots, \alpha_{N}\right]^{T}, I \subset R^{(N \times N)}$,

$Q=y_{i} y_{j} \varphi\left(x_{i}\right)^{T} \varphi\left(x_{j}\right)$. According to the Mercer condition, the kernel equation can be obtained

$$
K\left(x, x_{j}\right)=\phi(x)^{T} \cdot \phi\left(x_{j}\right)
$$

In LSSVM, quadratic programming problem is changed as the problem of solving linear equation groups, which simplifies the calculation quantity. At last, the decision function of the classifier of LS-SVM can be expressed as following:

$$
y(x)=\operatorname{sign}\left[\sum_{i=1}^{N} \alpha_{i} y_{i} \phi\left(x, x_{i}\right)+b\right]
$$

Where $\alpha_{i}$ and $b$ can be acquired in equation (5). In this paper, we have kernel functions in common use as follows:

$$
K\left(x_{i}, x_{j}\right)=\exp \left(-\left\|x_{i}-x_{j}\right\|^{2} / \sigma^{2}\right)
$$

The radial basis function [9-10] (RBF) is used as the kernel function of the LS-SVM because RBF kernel tends to give good performances under general smoothness assumptions.

B. The particle swarm optimization (PSO)

The particle swarm optimization [11] is proposed by Kennedy and Eberhart in 1995 and has been applied successfully to various optimization problems. The particle swarm optimization algorithm can complete optimization search by the collaboration and competition of the individuals in complex space. When solving optimization problem by using the PSO algorithm, each particle, that is to say the solution to the problem, is a point in the search space. Each particle has its own position vector and speed vector, which deciding its moving direction and distance. There is a fitness function which used to evaluate the superiority degree of each particle. All particles memorize and share the current optimal particle, and they will search in the search space until find the optimization solution. The iterative algorithm is used in the PSO algorithm. At each iteration, every particle closes up to two points in the search space at the same time. One point is its own optimum solution in the post search, and the other is the global optimum solution for all particles in historical search. In the course of search, every particle updates its position and speed as the follow formulas:

$$
\begin{gathered}
v_{i d}^{(t+1)}=\omega v_{i d}^{(t)}+c_{1} r_{1}\left(p_{i d}^{(t)}-x_{i d}^{(t)}\right)+c_{2} r_{2}\left(p_{g d}^{(t)}-x_{i d}^{(t)}\right) \\
x_{i d}^{(t+1)}=x_{i d}^{(t)}+v_{i d}^{(t+1)}
\end{gathered}
$$

Where $d=1,2, \cdots, n, i=1,2, \cdots, m, n$ is the dimension of the search space, and $m$ is population size; $x_{i d}^{t}$ is the current position of particle ${ }^{i}$ at a certain iteration $t ; v_{i d}^{t}$ is the velocity of particle $i$ at a certain iteration $t$, deciding the direction of $x_{i d}^{t+1} ; p_{i d}$ is the 
optimum solution of particle $i$ in the post search; $p_{g d}$ is the global optimum solution for all particles in historical search; $r_{1}$ and $r_{2}$ are random values between 0 and $1 ; c_{1}$ and $c_{2}$ are learning parameters, usually they are the constants in the range [0,2]; $\omega$ is the inertia weight, which can affect the searching ability. Usually, the inertia weight $\omega$ can be described by the equation (11)

$$
\omega(t)=\omega_{i n}-\frac{\omega_{i n}-\omega_{\text {end }}}{T_{\max }} \cdot t
$$

Where $\omega_{i n}$ is the original inertia weight value; $\omega_{\text {end }}$ is the inertia weight value at the most iterative time; $T_{\max }$ is the whole iterative time; $t$ is the current iterative time. According to the equation (12), the inertia weight $\omega$ will linearly decrease from $\omega_{i n}$ to $\omega_{\text {end }}$ with the evolution of iterative.

The research shows that the inertia weight value can decide the searching ability of the PSO algorithm. The large values of this parameter can increase its global searching ability, which will benefit to dap out from the local maximum value point but may not find the more accurate solutions; while the small values can increase the local searching ability, which will help for finding the accurate solutions but decreasing its convergence speed [11-12]. When increasing the iterative times and linearly decreasing the inertia weight value, we can acquire the optimization value with fast speed and high accuracy.

The fitness function can evaluate the objective values of all particles. The choice of the fitness function is very important because it is on this basis that the PSO evaluates the goodness of each particle solution for the LS-SVM classification system. In the PSO algorithm, the fitness function [3] as shown below:

$$
f\left(\sigma^{2}, \gamma\right)=\frac{\sum_{i=1}^{N} \operatorname{Inv}\left(a b s\left(\operatorname{sign}\left[\sum_{i=1}^{N} \alpha_{i} y_{i} \phi\left(x, x_{i}\right)+b\right]-y\right)\right)}{N}
$$

C. The PSO and LS-SVM Classification

Because the RBF function only has a standardization parameter $\sigma$ and calculates simply, we adopt RBF function as the kernel function. In LS-SVM model, the parameter $\gamma$ and parameter $\sigma$ of the RBF function are chosen according to experience. In this way, for different sample sets, the optimal parameter values are difficult to be found, which affects the fault diagnosis efficiency and accuracy. In the PSO and LS-SVM (PSOLSSVM) model, the kernel function parameters are selected by the PSO algorithm, which can find the optimal parameter value quickly. The process obtaining the parameters from PSO -LSSVM model [3] is as following:

Step1: Initialize the corresponding parameters, such as swarm of size $\mathrm{m}$, the parameter $(\sigma, \gamma)$, learning parameters $c_{1}$ and $c_{2}$, the inertia weight values $\omega_{i n}$ and $\omega_{\text {end }}$, the iterative time $T_{\max }$, initialize all particles with random positions and velocities;

Step2: Set the best position $\left(p_{i d}\right)$ of each particle equal to each particle current position, detect the best global position $\left(p_{g d}\right)$ and set $p_{g d}$ equal to the position of the best initial particle;

Step3: Train an LSSVM classifier and calculate the fitness function value of each particle;

Step4: Calculate the inertia weight value $\omega$ according to the equation (11), update the velocity and position of every particle according to the equations (9) and (10);

Step5: For each particle, compare its current value with $p_{i d}$ in history. If the current value is better, then set the current value as the new $p_{i d}$;

Step6: Establish the best objective value of the swarm. If the objective value is better than the value of $p_{g d}$ in history, then set the current value as the new $p_{g d}$; 
Step7: If one of the stopping conditions (reaching at the given fitness value or the maximal iteration time) is satisfied, then output $p_{g d}$; otherwise go to Step 2.

D. The Design of Detection System

According to the character of the video advertising, the video advertising test system based on the color histogram and PSO-LSSVM algorithm is been designed in this paper. The detection system can realize the function of the fast classification for the video advertising data. The flow chart of the detection system is shown in Figure 4.

As can be seen from the figure, the detection process is as follows: First, the relevant parameters are initialized, and select the video you want to analyze the data file; then using the color histogram of the lens edge detection method for video shot segmentation to achieve mutation detection lens; then and classify the camera lens to use the LS-SVM algorithm based on the data characteristics; Finally, precisely position the lens and merge them to get the final video advertising clips, enabling automatic quickly to locate video advertising segment.

Two indicators are adopted to evaluate video advertising performance: precision and recall; precision is used to measure the capacity of detection system excluding the unrelated video advertising. Recall is used to measure the capacity of detection system to retrieve relevant video advertising. These two indicators [8] is defined as follows:

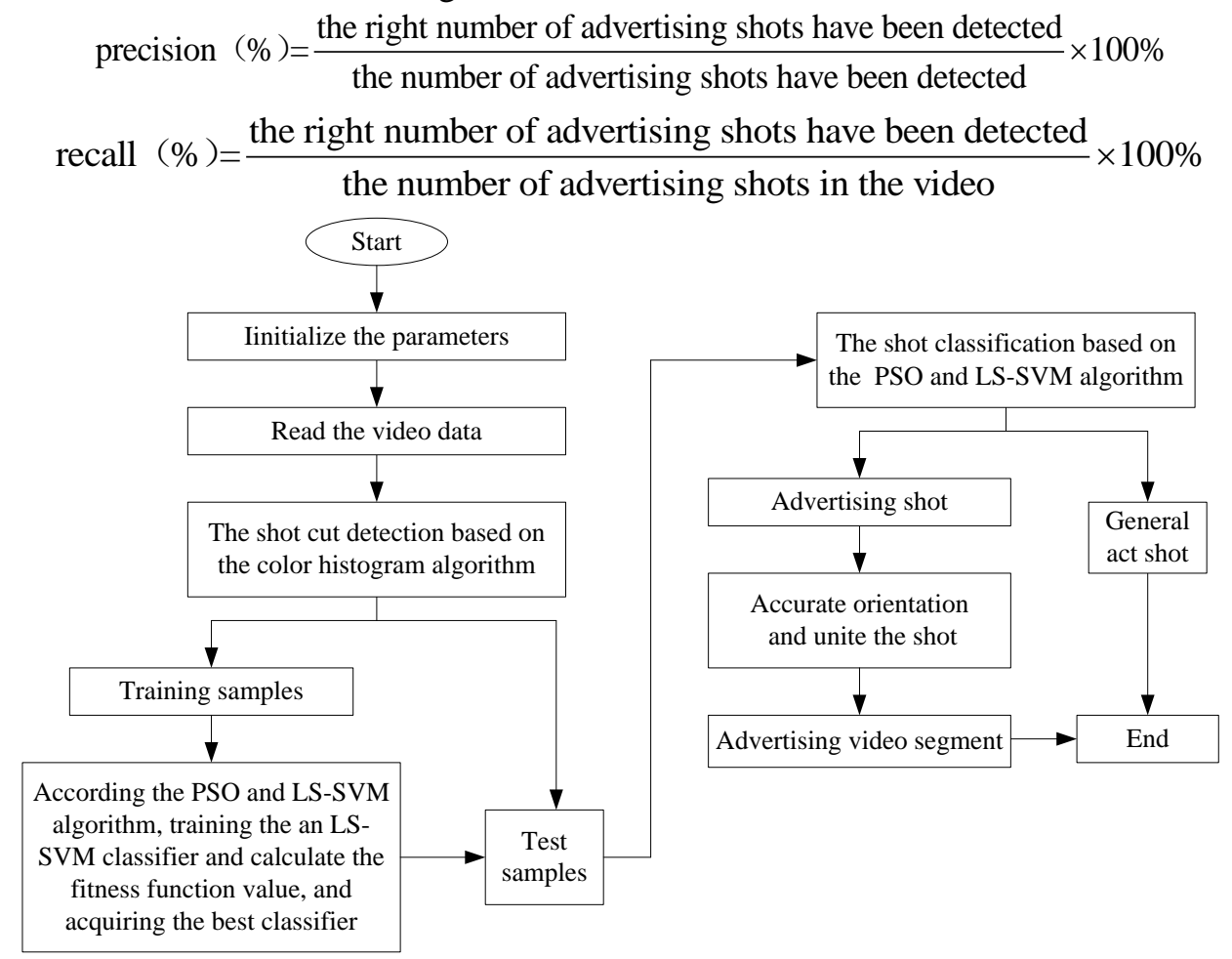

Figure 4. The Flow Chart of the Video Advertising Detection System

\section{The Experiment}

In order to verify the correctness of the detection method of video advertising in this paper, the 120 video segments of a local TV station were detected, which contains a total of 63 segments of television programs and 57 general advertising. The detection process as follow:

The video segments have been divided into two groups. The group 1 has included 55 video segments, which have made up of 32 segments of television programs and 23 segments general advertising. The total number of the advertising shot is 2328 . 
The group 2 has included 65 video segments, which have made up of 31 segments of television programs and 34 segments general advertising. The total number of the advertising shot is 3279 .

In the experiment, the scale factor radial basis functions were given $\sigma=0.4, C=500$.

In PSO-LSSVM method, the particle swarm searching parameters are set as: the swarm size is 50 , vector dimension is 3 , the learning parameters $c_{1}=c_{2}=2$, the original inertia weight $\omega_{i n}=0.9$, and the last inertia weight $\omega_{\text {end }}=0.4$, the other parameters $\alpha \in(0.01,10)$, $\gamma \in(0.01,50)$. In the LS-SVM method, the optimal parameter is chosen according to experience.

According to the group, the two groups have select different segments as training samples, and the residual segments as test samples. In group 1, select one of the 12 segments ordinary TV programs and 9 segments advertising as training samples, and the residual 34 segments for the test set.

In group 2, select one of the 17 segments ordinary TV programs and 13 segments advertising as training samples, and the residual 34 segments for the test set.

After testing, the test results have shown in Table 1.

\section{Table 1. The Detection Result Based on the Color Histogram and LS-SVM Algorithm}

\begin{tabular}{|c|c|c|c|c|c|}
\hline & $\begin{array}{c}\text { Ads. shot } \\
\text { number }\end{array}$ & $\begin{array}{c}\text { Detection ads. } \\
\text { shot number }\end{array}$ & $\begin{array}{c}\text { Correct ads. } \\
\text { shot number }\end{array}$ & Precision \% & Recall \% \\
\hline Group 1 & 2328 & 2419 & 2198 & 90.9 & 94.4 \\
\hline Group 2 & 3279 & 3401 & 3105 & 91.3 & 94.7 \\
\hline $\begin{array}{c}\text { Total } \\
\text { result }\end{array}$ & 5607 & 5820 & 5303 & 91.1 & 94.6 \\
\hline
\end{tabular}

In the group 1, the detection results as follow: the actual advertising shot number is 2328 , the detection advertising shot number is 2419 , the correct detection advertising shot number is 2198 , the precision is $90.9 \%$, and the recall rate is $94.4 \%$.

In the group 2, the detection results as follow: the actual advertising shot number is 3279 , the detection advertising shot number is 3401 , the correct detection advertising shot number is 3105 , the precision is $91.3 \%$, and the recall rate is $94.7 \%$.

In the total detection result, the actual advertising shot number is 5607 , the detection results as follow: the detection advertising shot number is 5820, the correct detection advertising shot number is 5303 , the precision is $91.1 \%$, and the recall rate is $94.6 \%$.

As can be seen from Table I , the test results of the video advertising detection based on the color histogram and LS-SVM algorithm have very high. The precision and the recall rate are over $90 \%$.

In order to verify the detection performance has been increased by using the PSOLSSVM algorithm. In the experiment, the same sample has been detected by using the PSO-LSSVM method and the LSSVM method. the test results have shown in Table 2.

Comparing the classification precision and recall of the two methods, the precision and recall of the video advertising detection adopting LSSVM method is 91.5\% and 94.6\%, while the precision and recall adopting PSO-LSSVM method is $93.4 \%$ and $97.5 \%$. The more experiments have been completed, the average precision and recall of advertising detection by using the LSSVM method is $91.2 \%$ and $94.5 \%$, while adopting the PSOLSSVM method is $93.1 \%$ and $97.2 \%$. 
Table 2. The Detection Result Based on the LS-SVM and PSO- LSSVM Algorithm

\begin{tabular}{|c|c|c|c|c|c|}
\hline $\begin{array}{c}\text { Detection } \\
\text { result }\end{array}$ & $\begin{array}{c}\text { Ads. shot } \\
\text { number }\end{array}$ & $\begin{array}{c}\text { Detection ads. } \\
\text { shot number }\end{array}$ & $\begin{array}{c}\text { Correct ads. } \\
\text { shot number }\end{array}$ & Precision \% & Recall \% \\
\hline LS-SVM & 2800 & 2895 & 2649 & 91.5 & 94.6 \\
\hline PSO-LSSVM & 2800 & 2923 & 2730 & 93.4 & 97.5 \\
\hline $\begin{array}{c}\text { Average result } \\
\text { (LS-SVM) }\end{array}$ & & & & 91.2 & 94.5 \\
\hline $\begin{array}{c}\text { Average result } \\
\text { (PSO- } \\
\text { LSSVM) }\end{array}$ & & & 93.1 & 97.2 \\
\hline
\end{tabular}

In the experiment, the arbitrary choice of a set of video data has been detected by using the two methods. The classification charts are shown in Figure 5 and Figure 6. The classification result based on LS-SVM is shown in Figure 5, and adopting the PSOLSSVM algorithm is shown in Figure 6. Obviously, the detection result in Figure 6 is better than that in Figure 5.

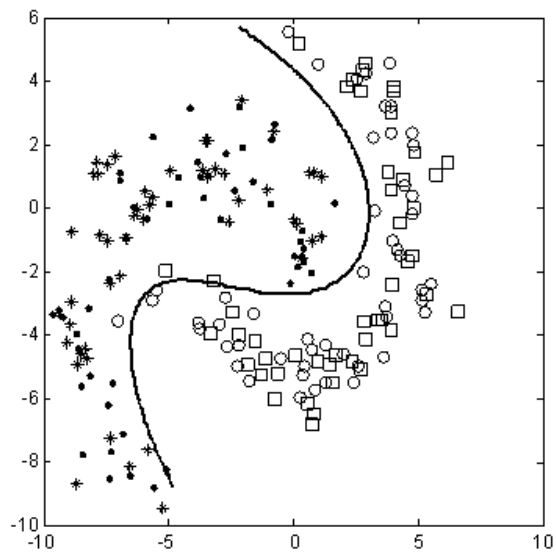

Figure 5. The Classification Result Based on the LS-SVM Algorithm

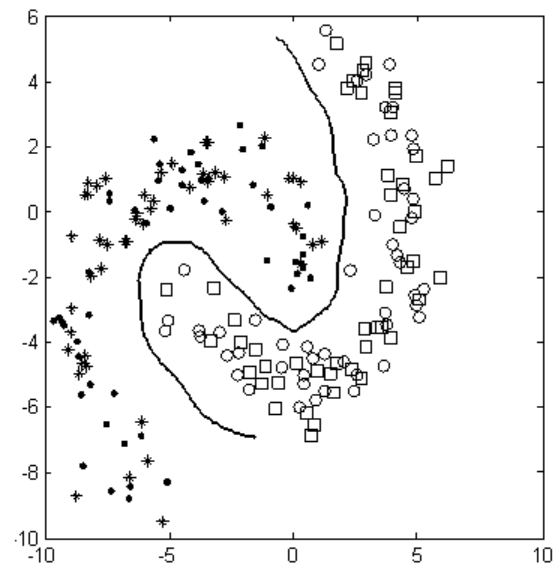

Figure 6. The Classification Result Based on the PSO-LSSVM Algorithm

The experiment results show that the classification precision and recall of the video advertising detection adopting the PSO-LSSVM method is higher than the LSSVM method. So, the method can realize accurate classification and detection of the detected video advertising. 


\section{Conclusion}

In this paper, propose the video advertising detection system based on the color histogram and LS-SVM algorithm .First shot segmentation method using color histogram, and then use the LS-SVM classifier to classify the lens, and then achieve accurate testing of video advertising The system uses a least squares support vector machine for classification, to improve the efficiency of the system to distinguish, not only can improve the efficiency of the relevant employees, but also to provide a reference for relevant teaching and research staff.

\section{References}

[1] Zhang L., Zhu Z., Zhao Y. and Lu H., "Video commercial detection based on the robustness of shot," CAAI Transactions on Intelligent Systems, vol. 2 no. 2, (2007), pp. 83-88.

[2] Li S. J., Ruan X. Z., Feng J. and Lin L., "A Novel Approach to Video Transition Detection Based on Machine Learning," Journal of Guangxi Normal University: Natural Science Edition, vol. 26 no. 1, (2008), pp. 228-231.

[3] Miene A., Dammeyer A. and Hermes T. H., "Advanced and adaptive shot boundary detection," Proc. of ECDL, vol. 8, (2001), pp. $39-43$.

[4] Huamin F., Wei F., Sen L. and Yong F., "A new general framework for shot boundary detection and key-flame extraction"Nov. 2005 Proceedings of the 7m ACM SIGMM international workshopœMultimedia information retrieval, (2005), pp. 121-126.

[5] Ohta Y., Kanade T. and Sakai T., "Color information for region segmentation," Computer Graphics and Image Processing, vol. 13 no. 3, (1980), pp. 222-241.

[6] Liu H. and Yu L., "Toward Integrating Feature Selection Algorithms for Classification and Clustering," IEEE. Transactions on Knowledge and Data Engineering, vol. 17 no. 5, (2005), pp. 491-502.

[7] Cao J. R. and Cai A.N., "A robust shot transition detection method based on support vector machine in compressed domain," Pattern Recogn Lett, vol. 28 no. 12, (2007), pp. 1534-1540.

[8] Liu N., Zhao Y. and Zhu Z., "Video Commercial Text Detection Based on Co-Training Strategy," Journal of Beijing Jiaotong University, vol. 34 no. 5, (2010), pp. 1-7.

[9] Osuna E., Freund R. and Girosi F., "An improved training algorithm for support vector machine," Proc. IEEE Workshop on Neural Networks for Signal Processing, Amelia Island, FL, (1997), pp. 276-285.

[10] Camps V. G., Martin G. J. and Rojo A. J., "Fuzzy sigmoid kernel for support vector classifiers," Neurocomputing, vol. 62, (2004), pp. 501-506.

[11] Kennedy J. and Eberhart R. C., "Particle swarm optimization," Proc of IEEE International Conference on Neural Networks, (1995), pp. 1942-1948.

[12] Liu H. and Yu L., "Toward Integrating Feature Selection Algorithms for Classification and Clustering," IEEE. Transactions on Knowledge and Data Engineering, vol. 17 no. 5, (2005), pp. 491- 502.

\section{Author}

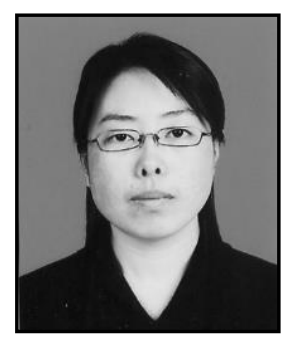

Xiao-ling Lan, she is a Lecturer, a Master, work in the Art college of Ludong University. Now, mainly work in the ADS. Her interests include data processing and multimedia technology field. 
International Journal of Multimedia and Ubiquitous Engineering

Vol.10, No.7 (2015) 